ARTICLE

https://doi.org/10.1038/s41467-019-13488-5

\title{
Self-organized twist-heterostructures via aligned van der Waals epitaxy and solid-state transformations
}

\author{
Peter Sutter (10 ${ }^{1 \star}$, Rina Ibragimova (i) ${ }^{2}$, Hannu-Pekka Komsa (i) ${ }^{2}$, Bruce A. Parkinson ${ }^{3}$ \& Eli Sutter (i) ${ }^{4}$
}

Vertical van der Waals (vdW) heterostructures of 2D crystals with defined interlayer twist are of interest for band-structure engineering via twist moiré superlattice potentials. To date, twist-heterostructures have been realized by micromechanical stacking. Direct synthesis is hindered by the tendency toward equilibrium stacking without interlayer twist. Here, we demonstrate that growing a $2 \mathrm{D}$ crystal with fixed azimuthal alignment to the substrate followed by transformation of this intermediate enables a potentially scalable synthesis of twisted heterostructures. Microscopy during growth of ultrathin orthorhombic SnS on trigonal $\mathrm{SnS}_{2}$ shows that vdW epitaxy yields azimuthal order even for non-isotypic 2D crystals. Excess sulfur drives a spontaneous transformation of the few-layer $\mathrm{SnS}$ to $\mathrm{SnS}_{2}$, whose orientation - rotated $30^{\circ}$ against the underlying $\mathrm{SnS}_{2}$ crystal - is defined by the SnS intermediate rather than the substrate. Preferential nucleation of additional SnS on such twisted domains repeats the process, promising the realization of complex twisted stacks by bottom-up synthesis.

\footnotetext{
${ }^{1}$ Department of Electrical \& Computer Engineering, University of Nebraska-Lincoln, Lincoln, NE 68588, United States. ${ }^{2}$ Department of Applied Physics, Aalto University, P.O. Box 11100, Fl-00076 Aalto, Finland. ${ }^{3}$ Department of Chemistry and School of Energy Resources, University of Wyoming, Laramie, WY 82071, United States. ${ }^{4}$ Department of Mechanical \& Materials Engineering, University of Nebraska-Lincoln, Lincoln, NE 68588, United States.

*email: psutter@unl.edu
} 
$\mathrm{V}$ an der Waals (vdW) heterostructures promise functional materials by integration of two-dimensional (2D) crystal building blocks ${ }^{1}$ while largely bypassing conventional structure- and lattice-matching requirements ${ }^{2,3}$. Recently, vertical heterostructures with controlled interlayer twist-a relative inplane (azimuthal) rotation of one 2D crystal against the otherhave attracted particular interest due to emerging electronic phenomena that are defined both by the atomic lattices and a periodic twist-moiré superlattice with substantially larger unit cell. For bilayer graphene with small magic-angle misorientation, this competition causes the emergence of electronic correlations and unconventional superconductivity ${ }^{4,5}$. In $2 \mathrm{D}$ semiconductor heterostructures, the interlayer coupling, electronic band structure, and optoelectronic properties depend on the twist angle $e^{6-12}$.

Mechanical stacking ${ }^{13-15}$ has been the preferred approach for fabricating vdW heterostructures ${ }^{6,7}$, as well as stacks with controlled static $^{16-18}$ or dynamically adjustable interlayer twist ${ }^{19}$. But factors such as a lack of scalability as well as concerns about uniformity and possible interface contamination are motivating a search for bottom-up synthesis methods for twisted vdW heterostructures. There are two fundamentally interesting regimes for which synthesis approaches need to be developed: (i) Precise small-angle interlayer twists, which cause moiré patterns that give rise to a modulation of the electronic structure within large unit cells whose lateral dimensions can exceed $100 \mathrm{~nm}$; and (ii) high-angle twists. In both cases, the realization of a controlled interlayer orientation during growth is hindered by the strong tendency of $2 \mathrm{D}$ crystals to stack in their equilibrium registry, i.e., azimuthally aligned without any twist, both for homo-stacks of the same or hetero-stacks of different but isostructural 2D materials ${ }^{20-29}$. The affinity toward equilibrium stacking makes it particularly challenging to realize small interlayer twist angles. In recent work, we showed that architectures that depart from the usual 2D vdW heterostructure geometry-specifically layered nanowires with Eshelby twist-can spontaneously yield selforganized twist moirés with small twist angles that are tunable via the nanowire diameter ${ }^{30}$.

For larger interlayer twists, the weak interaction between layered crystals can enable a different avenue toward the bottomup synthesis of twist heterostructures via a two-step process in which the synthesis of an intermediate 2D (or 3D) crystalline phase $(B)$ on a layered substrate $(A)$ is followed by the conversion of the intermediate to a final phase $A^{\text {rot }}$ with a defined azimuthal rotation relative to the substrate. Such a two-step process has recently been used for the creation of a dodecagonal quasicrystal in $30^{\circ}$ twisted bilayer graphene ${ }^{31}$. In this case, monolayer h-BN served as an intermediate $2 \mathrm{D}$ crystal that grows on epitaxial graphene/SiC under $30^{\circ}$ rotation, and a high-temperature annealing step replaced this template by a graphene layer with the same orientation, i.e., $30^{\circ}$ twist relative to the underlying graphene. While this demonstration was based on a substitution between isostructural $2 \mathrm{D}$ honeycomb crystals, a similar strategy could be extended to other 2D/layered materials, such as metal chalcogenide semiconductors as well as intermediates that are not isotypic with the substrate. The viability of this generalized approach depends on two key requirements: Growth of the intermediate $B$ has to involve a fixed azimuthal registry of $B$ relative to the substrate $A$; and in the final solid-state transformation $B \rightarrow$ (twisted) $A^{\text {rot }}$, the azimuthal orientation of the resulting crystal should be determined by the lattice structure of the intermediate rather than the substrate.

Here, we demonstrate the realization of this concept for layered tin chalcogenide semiconductors. For these materials, several stable layered crystal phases exist with different chalcogen content and prior work has demonstrated the conversion from chalcogen-rich trigonal $\mathrm{SnS}(\mathrm{e})_{2}$ dichalcogenide phases to orthorhombic SnS(e) monochalcogenides by generation of chalcogen vacancies, either thermally $^{32}$, by electron irradiation ${ }^{33}$, or plasma exposure ${ }^{34}$. For the bottom-up growth of twisted vdW heterostructures, a bulk $\mathrm{SnS}_{2}$ single crystal plays the role of the layered substrate, $A$. The intermediate $2 \mathrm{D}$ crystal phase, $B$, is ultrathin $\mathrm{SnS}$ grown by vdW epitaxy. In situ low-energy electron microscopy (LEEM) during growth allows us to identify the sequence of azimuthally aligned vdW epitaxy and spontaneous solid-state transformations that ultimately gives rise to self-organized $\mathrm{SnS}_{2}$ twist heterostructures and incipient complex architectures, such as periodic vertical stacks with multiple twisted vdW interfaces.

\section{Results and discussion}

$\mathrm{SnS}$ was evaporated by congruent sublimation of intact formula units (i.e., $\mathrm{SnS}$ molecules) from a stoichiometric SnS powder precursor $^{23}$ onto freshly cleaved $\mathrm{SnS}_{2}$ single crystals (and other substrates, see below) while imaging the growth process in real time by LEEM. At temperatures below $280^{\circ} \mathrm{C}$ and between $320-340^{\circ} \mathrm{C}$, nucleation and growth produces heterostructures of single-crystalline few-layer SnS with lateral size up to several $\mu \mathrm{m}$ on atomically flat $\mathrm{SnS}_{2}$ vdW substrates (Fig. 1a). Note that this geometry is the inverse of previously reported $\mathrm{SnS}_{2} / \mathrm{SnS}$ vertical heterostructures ${ }^{35}$. Selected-area low-energy electron diffraction (micro-LEED) was used to analyze the crystal structure and lattice registry. The substrate shows the hexagonal surface mesh of single-crystalline $\mathrm{SnS}_{2}$ (Fig. 1b) with two distinct sets of alternating intense and weaker diffraction spots, associated with $(10)^{\mathrm{SnS}_{2}}$ and $(01)^{\mathrm{SnS}_{2}}$ reciprocal lattice vectors ${ }^{36}$. Composite micro-LEED patterns of SnS domains and of the surrounding $\mathrm{SnS}_{2}$ (Fig. 1c) show monocrystalline few-layer SnS. In contrast to other substrates (graphite, graphene) ${ }^{23}$ where $\mathrm{SnS}$ grows with random orientation, vdW epitaxy on $\mathrm{SnS}_{2}$ locks the $\mathrm{SnS}$ domains into a well-defined azimuthal alignment, found in all heterostructures reported here. In reciprocal space, the azimuth of the $(11)^{\mathrm{SnS}}$ reflection of $\mathrm{SnS}$ aligns with the $(10)^{\mathrm{SnS}_{2}}$ reflection of $\mathrm{SnS}_{2}$. Consistent with a relatively weak interlayer interaction, which can lock the SnS layer into a well-defined azimuthal orientation with the underlying $\mathrm{SnS}_{2}$ lattice but is not strong enough to force lattice matching, there is no detectable strain and the measured in-plane lattice constants of $\mathrm{SnS}$ and $\mathrm{SnS}_{2}$ coincide with values reported for the respective bulk single crystals ${ }^{37,38}$. Figure $1 \mathrm{~d}$, e illustrate the observed real-space azimuthal alignment in the SnS/ $\mathrm{SnS}_{2}$ heterostructures, where the projected $\mathrm{Sn}-\mathrm{S}$ bonds in $\mathrm{SnS}$ align with one of three bond directions of the $\mathrm{SnS}_{2}$ lattice. This preferred orientation generates three possible SnS domain orientations, separated by $120^{\circ}$ (see Supplementary Fig. 2). The growth process shown in Fig. 1 can be rationalized by densityfunctional theory (DFT) based nudged elastic band calculations (Supplementary Fig. 3). The DFT results support a picture in which SnS congruently sublimed from precursor powder adsorbs on the $\mathrm{SnS}_{2}$ surface, diffuses, and nucleates or is incorporated into $\mathrm{SnS}$ domains. On-surface $\mathrm{SnS}$ dissociation is inhibited by large energy barriers (close to $1 \mathrm{eV}$ ), while lower activation energies $(0.2-0.4 \mathrm{eV})$ enable the facile $\mathrm{SnS}$ surface diffusion by rotation between different $\mathrm{S}$-sites.

Atomic force microscopy (AFM) has been used to further analyze the vertical tin chalcogenide heterostructures. Figure 2 shows AFM images of the $\mathrm{SnS}_{2}$ substrate prior to $\mathrm{SnS}$ growth, and of a $\mathrm{SnS} / \mathrm{SnS}_{2}$ heterostructure. The freshly cleaved $\mathrm{SnS}_{2}$ substrate is flat, with atomic terraces separated by single-layer high steps (Fig. 2a). Following SnS deposition, AFM shows ultrathin fewlayer SnS domains with lateral dimensions up to several $\mu \mathrm{m}$, consistent with the LEEM results of Fig. 1 and evidence that synthesis on $\mathrm{SnS}_{2}$ avoids the strong tendency toward vertical growth of thicker SnS found for other vdW substrates ${ }^{23}$. The 
a

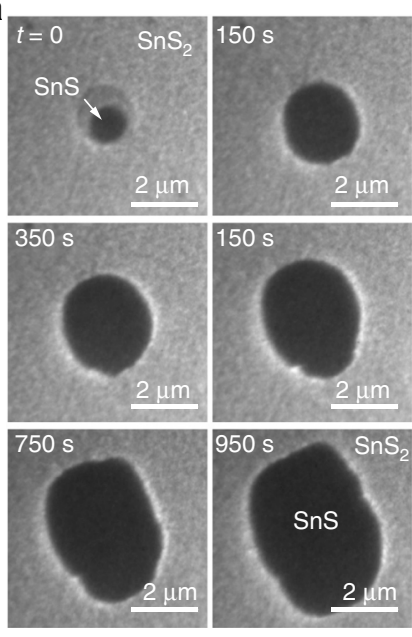

b

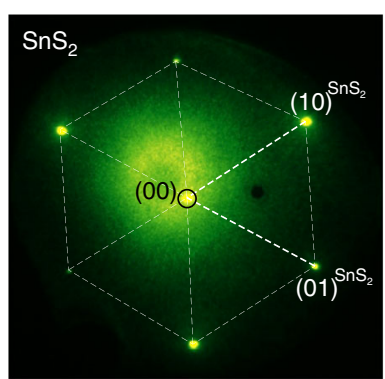

d

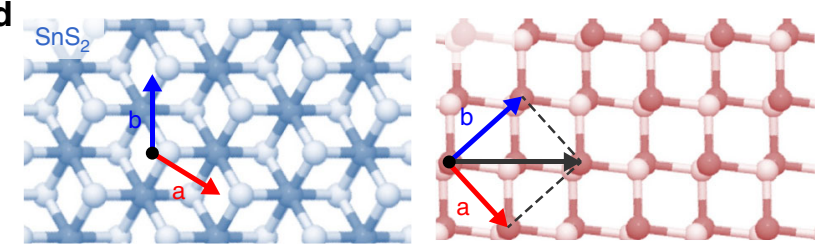

e

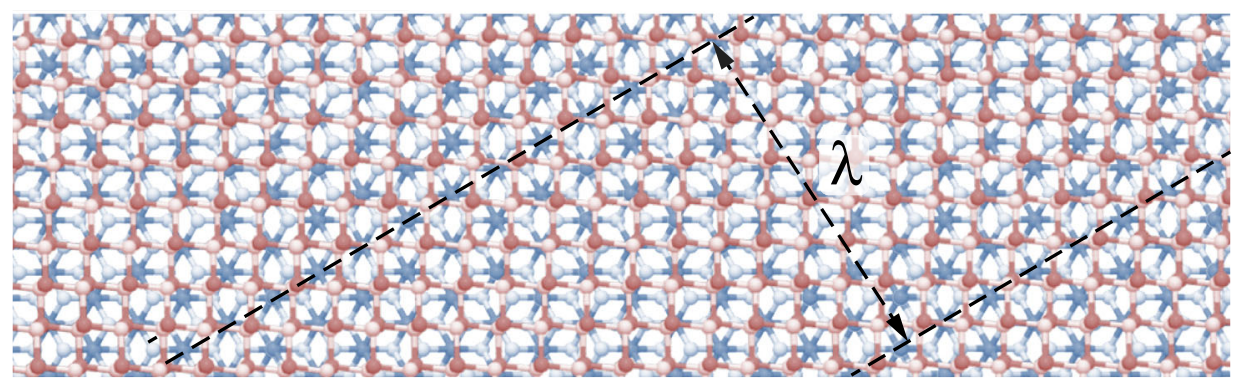

Fig. 1 Direct growth of $\mathrm{SnS} / \mathrm{SnS}_{2}$ heterostructures at $T=320^{\circ} \mathrm{C}$. a Real-time image sequence during deposition of $\mathrm{SnS}$ onto single-crystalline $\mathrm{SnS}$. Imaging electron energy: $E=4.3 \mathrm{eV}$. b Micro-LEED pattern of the $\mathrm{SnS}_{2}$ substrate $(E=50 \mathrm{eV})$. c Micro-LEED pattern of the $\mathrm{SnS} / \mathrm{SnS} 2 \mathrm{heterostructure} \mathrm{(E}=$ $50 \mathrm{eV})$. Note the azimuthal alignment between the $(11)^{\mathrm{SnS}}$ reflection of $\mathrm{SnS}$ and the $(10)^{\mathrm{SnS}_{2}}$ reflection of $\mathrm{SnS}_{2}$. The measured lattice mismatch along this direction is 8\%; both lattices adopt their bulk lattice constants during the vdW epitaxy. $\mathbf{d}$ Models of the SnS 2 and SnS lattices with the observed azimuthal alignment. e Overlay of the two structures. $\lambda$ denotes the wavelength of the stripe moiré pattern generated between the SnS and $\mathrm{SnS}_{2}$ lattices (see also Supplementary Fig. 1) 48

domain shown in Fig. 2b, for example, varies in thickness between $3-4$ SnS layers $\left(1 \mathrm{~L}^{\mathrm{SnS}} \approx 0.56 \mathrm{~nm}\right)^{38}$ and its vdW interface lies $1 \mathrm{~L}^{\mathrm{SnS}_{2}}$ below the average substrate surface. SnS flakes in this thickness range should allow the experimental realization of phenomena such as in-plane ferroelectric ordering ${ }^{39-41}$ and photostriction $^{42}$ predicted for few-layer group IV monochalcogenides with odd number of layers. Surface potential measurements using Kelvin probe force microscopy (KPFM, see below) indeed show clear thickness-dependent properties (Fig. 2c, Supplementary Fig. 4). Such ultrathin SnS domains generally crystallize in a rounded shape bounded by micro-facets ${ }^{43}$. Also evident is the transformation of the atomically flat $\mathrm{SnS}_{2}$ surface into a patchwork of single-layer deep pits, where $\mathrm{SnS}_{2}$ was apparently removed during SnS growth (Fig. 2b, inset). Analysis shows that these single-layer deep vacancy islands cover about $20 \%$ of the $\mathrm{SnS}_{2}$ surface after $\mathrm{SnS}$ growth (see Supplementary Fig. 5). Comparison with the step orientation in the $\mathrm{SnS}_{2}$ pits, along with the azimuthal orientation determined by micro-LEED, identifies the SnS edges as majority (110) and (110) facets, complemented by smaller segments of vicinal (100) and (010) facets (Fig. 2d, e). This domain shape is consistent with a recent analysis of kinetic growth shapes of thin $\mathrm{SnS}$ flakes ${ }^{44}$.

Whereas high and low substrate temperatures ( $T$ ) favor direct growth of azimuthally aligned $\mathrm{SnS} / \mathrm{SnS}_{2}$ heterostructures, intermediate $T \sim 300{ }^{\circ} \mathrm{C}$ promotes an entirely different behavior, analyzed via real-time microscopy and diffraction (Figs. 3, 4). The initial nucleation and growth, identical to that shown in Fig. 1, again produces $\mu \mathrm{m}$-sized $\mathrm{SnS}$ domains (Fig. 3; $0 \leq t \leq 70 \mathrm{~s}$ ).
Beginning at $t=80 \mathrm{~s}$, the uniform contrast characteristic of $\mathrm{SnS}$ changes and a brighter phase (labeled $\mathrm{t}-\mathrm{SnS}_{2}$ ) appears and progressively spreads across the entire domain (80-110 s), transforming the previously rounded $\mathrm{SnS}$ domain into a shape with extended straight facets (110-130 s). Repeated experiments with different growth conditions show no clear correlation of this transformation process with temperature (within the stated window, i.e., $280-320^{\circ} \mathrm{C}$ ), size of the SnS intermediate, or any features of the underlying substrate.

The product of the transformation is identified in Fig. 4. Imaging at different electron energy, $E$, shows the $\mathrm{SnS}_{2}$ substrate coexisting with two types of $\mu \mathrm{m}$-scale domains with different $E$ dependent contrast (Fig. 4a). The analysis of these phases by micro-diffraction is shown in Fig. $4 \mathrm{~b}-\mathrm{e}$. The diffraction patterns of hexagonal $\mathrm{SnS}_{2}$ (Fig. 4c) and orthorhombic $\mathrm{SnS}$ (Fig. 4d) are identical to Fig. 1, including the fixed azimuthal alignment of SnS on $\mathrm{SnS}_{2}$. Figure $4 \mathrm{e}$ shows diffraction from one of the domains transformed from SnS. The pattern is a superposition of two sets of hexagonal reflections, rotated relative to each other by $30^{\circ}$. From this result, we conclude that the converted domains consist of twisted $\mathrm{t}-\mathrm{SnS}_{2}$, rotated in-plane by $30^{\circ}$ relative to the substrate lattice; and the $\mathrm{t}-\mathrm{SnS}_{2}$ is ultrathin, so that it contributes jointly with the underlying $\mathrm{SnS}_{2}$ to surface-sensitive diffraction. A further diffraction analysis provides evidence for superlattice reflections that arise from the twist moiré pattern of the hexagonal $\mathrm{SnS}_{2}$ crystals (Supplementary Fig. 6). Micro-LEED patterns on domains of $30^{\circ}$ twisted $\mathrm{t}-\mathrm{SnS}_{2} / \mathrm{SnS}_{2}$ show superlattice spots centered around the zone center and the first-order 

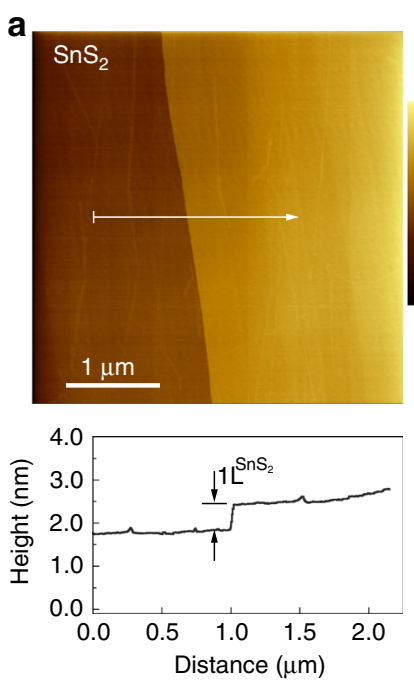
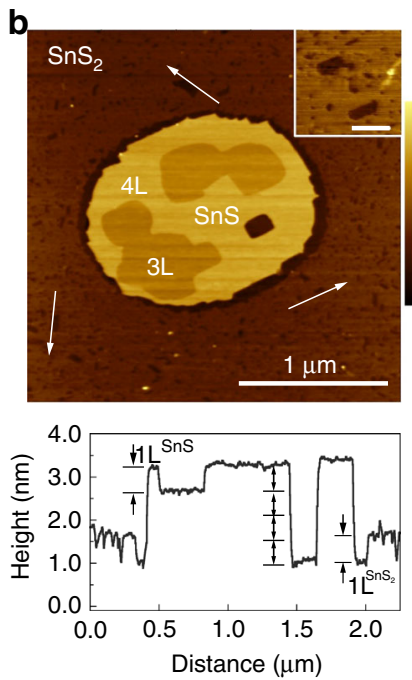

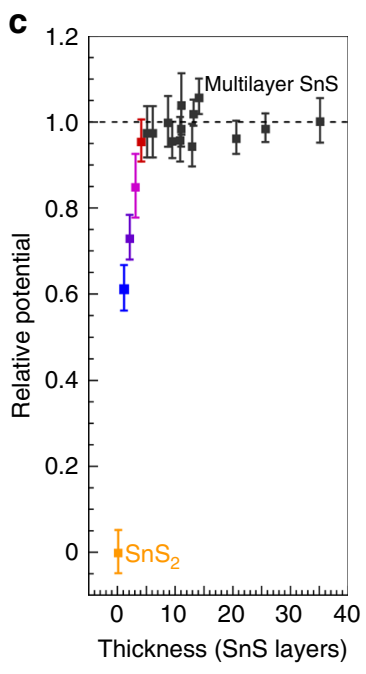

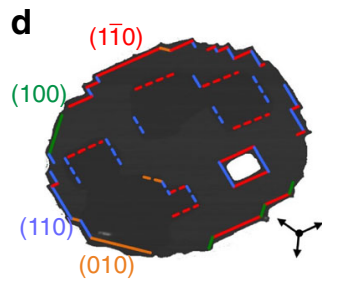

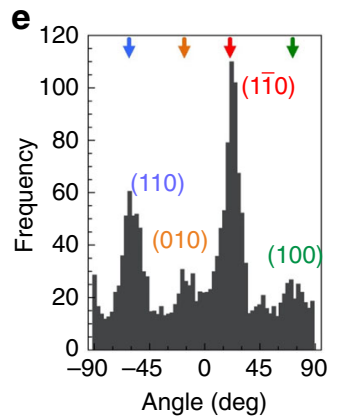

Fig. 2 AFM of single-crystalline $\mathrm{SnS}_{2}$ and ultrathin $\mathrm{SnS} / \mathrm{SnS}_{2}$. a AFM image of the $\mathrm{SnS}_{2}$ substrate with a monoatomic step. Bottom: Height profile along the line marked in the image. The step has height $1 \mathrm{~L}^{\mathrm{SnS}_{2}} \approx 0.6 \mathrm{~nm}$. Color scale: $3 \mathrm{~nm}$. b AFM image of an ultrathin SnS/SnS $\mathrm{S}_{2}$ vertical heterostructure. Note the abundant single-layer deep vacancy islands on the $\mathrm{SnS}_{2}$ surface, shown magnified in the inset (inset scale bar: $100 \mathrm{~nm}$ ). Bottom: Height profile along the line marked in the image. Since the $\mathrm{SnS} / \mathrm{SnS}_{2} v d W$ interface lies $1 \mathrm{~L}^{\mathrm{SnS}_{2}}$ below the average substrate surface, this particular $\mathrm{SnS}$ domain varies in height between $3-4 \mathrm{~L}^{\mathrm{SnS}}$. Color scale: $4 \mathrm{~nm}$. c Kelvin probe force microscopy potential measurements of few-layer $\mathrm{SnS}$ relative to the $\mathrm{SnS} \mathrm{S}_{2}$ substrate, normalized to the potential of thicker (multilayer) SnS (see Supplementary Fig. 4). Error bars represent the full width at half maximum of Lorentzian fits to the distribution of surface potentials in areas of contstant SnS thickness. d Footprint of the SnS island shown in c. with color-coded edge facet segments. Arrows indicate the edge orientations of $\mathrm{SnS}_{2}$ vacancy islands marked in $\mathbf{b}$. e Histogram of facet orientations determined from the AFM image shown in $\mathbf{b}$. Source data are provided as a Source Data file.
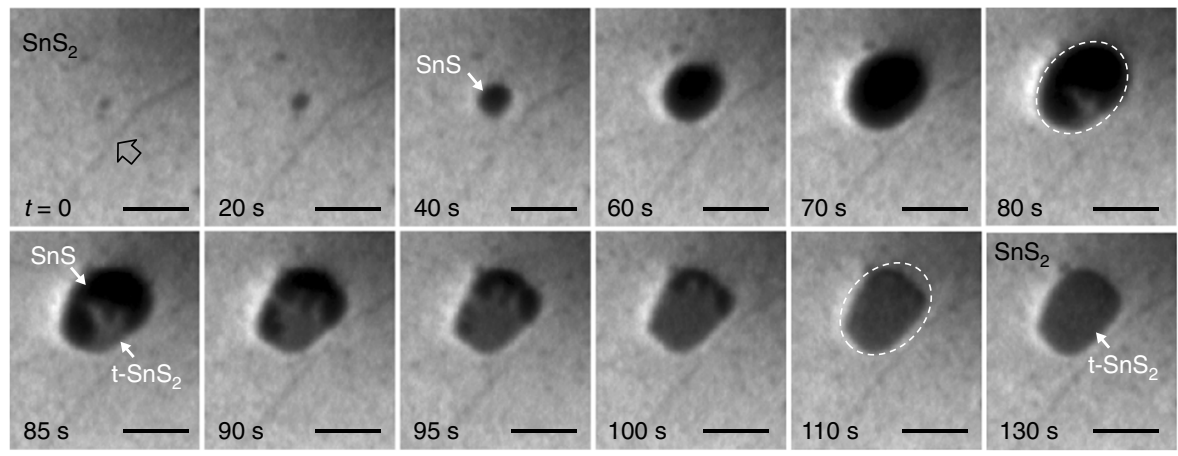

Fig. 3 Phase conversion in $\mathrm{SnS} / \mathrm{SnS}_{2}$ heterostructure growth at $T=300^{\circ} \mathrm{C}$. Real-time image sequence during deposition of SnS onto single-crystalline $\mathrm{SnS}_{2}$. The arrow at $t=0$ marks a surface step on the $\mathrm{SnS}_{2}$ substrate. Transformation from SnS to t-SnS 2 starts at $t=80 \mathrm{~s}$. Note that the precise shape of the $\mathrm{SnS}$ flakes is difficult to image due to their large $(\sim 1 \mathrm{eV})$ difference in surface potential relative to the surrounding $\mathrm{SnS}_{2}$ surface and the resulting strong electric fields near the $\mathrm{SnS}$ edge. $E=5.0 \mathrm{eV}$. Scale bar: $1 \mu \mathrm{m}$.

reflections of $\mathrm{t}-\mathrm{SnS}_{2}$ (Supplementary Fig. 6a-d). Fast-Fourier transforms of such diffraction patterns reflect the emerging dodecahedral structural motifs associated with a Stampfli-tiling quasicrystal ${ }^{45}$ (Supplementary Fig. 6e, f) as realized recently in $30^{\circ}$ twisted bilayer graphene ${ }^{31}$. The mechanism identified here, involving a solid-state transformation of a $\mathrm{SnS}$ intermediate, can also explain previously observed moiré patterns in $\mathrm{SnS}_{2}$ crystals synthesized by co-evaporation of $\mathrm{Sn}$ and $\mathrm{S}^{46}$. Consistent with previous work on twisted bilayer $\operatorname{MoS}_{2}{ }^{7}$, calculations show modifications of several key properties for $30^{\circ}$ twisted $\mathrm{SnS}_{2}$. The twisted vdW gap of $6.22 \AA$ is much larger than in equilibriumstacked bilayer $(5.87 \AA)$ or bulk $(5.82 \AA) \mathrm{SnS}_{2}$, and the interlayer binding energy decreases from $0.22 \mathrm{eV} /$ unit cell in the aligned bilayer to $0.16 \mathrm{eV} / \mathrm{unit}$ cell in the twisted bilayer. Electronically, the twist decouples the layers at the vdW gap in $\mathrm{t}-\mathrm{SnS}_{2}$ so that in a twisted bilayer they behave much like two monolayers, except for a few states around the $\Gamma$-point (Fig. $4 \mathrm{~g}$ ).
Evidently, a source of sulfur is required for the transformation of SnS to t-SnS ${ }_{2}$. The large SnS bond dissociation energy $(467 \mathrm{~kJ}$ mol $\left.^{-1}\right)^{47}$, congruent SnS sublimation ${ }^{23}$, and large on-surface dissociation energy (Supplementary Fig. 3; Supplementary Table 1) rule out SnS dissociation as the primary source of $S$, which instead comes from the slow thermal decomposition of the $\mathrm{SnS}_{2}$ substrate, explaining the formation of vacancy islands on the $\mathrm{SnS}_{2}$ surface (Fig. 2). An alternative source of excess sulfur is the presence of S-rich minority phases in the nominally pure SnS precursor powder, shown in recent work to spontaneously produce layered $\mathrm{SnS}-\mathrm{SnS}_{2}$ core-shell heterostructures on mica vdW substrates ${ }^{48}$. Both the release of $\mathrm{S}$ from the $\mathrm{SnS}_{2}$ substrate and the supply of excess $S$ from the precursor are consistent with the limited $T$-range in which the spontaneous $\mathrm{SnS} \rightarrow \mathrm{t}-\mathrm{SnS}_{2}$ conversion is observed, as supported by DFT (Supplementary Fig. 3, Supplementary Fig. 7, Supplementary Fig. 8, Supplementary Table 2). At lower $T$, the thermal decomposition $\mathrm{SnS}_{2} \rightarrow \mathrm{SnS}+\mathrm{S}$ 

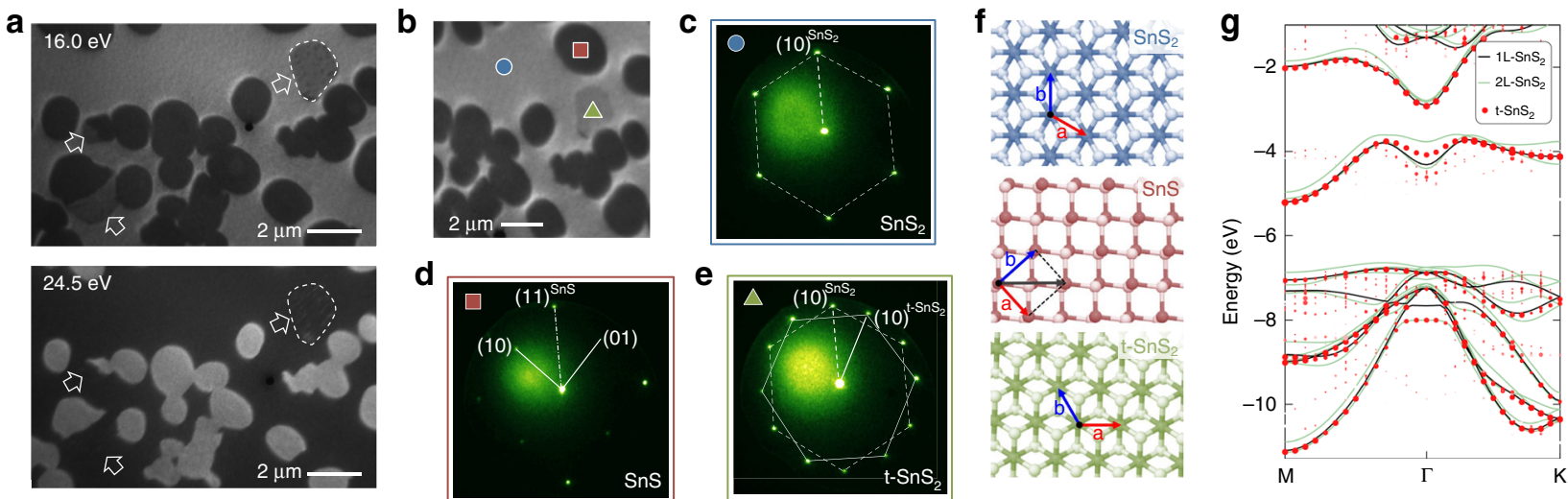

Fig. 4 Identification of the phases obtained in $\mathrm{SnS} / \mathrm{SnS}_{2}$ heterostructure growth at $T=300^{\circ} \mathrm{C}$. a LEEM image at $16.0 \mathrm{eV}$ electron energy (top), and same sample area imaged at $24.5 \mathrm{eV}$ electron energy (bottom). $\mathbf{b}$ LEEM image at $16.0 \mathrm{eV}$, showing the three different phases involved in growth at intermediate temperature $\left(T \sim 300^{\circ} \mathrm{C}\right)$. c-e Micro-LEED patterns obtained from the three distinct phases marked in b. c. SnS ${ }_{2}$ substrate; $\mathbf{d}$ Single-crystalline SnS:

e Ultrathin twisted t-SnS 2 , rotated $\sim 30^{\circ}$ relative to the $\mathrm{SnS}_{2}$ substrate. $\mathbf{f}$ Top-views of the three structures, namely $\mathrm{SnS} \mathrm{S}_{2}$ (top), $\mathrm{SnS}_{\text {aligned }}$ with the $\mathrm{SnS} \mathrm{S}_{2}$ substrate (center), and twisted t- $\mathrm{SnS}_{2}$, rotated $\sim 30^{\circ}$ relative to the $\mathrm{SnS}_{2}$ substrate (bottom). $\mathbf{g}$ Calculated band structures of single- and bilayer $\mathrm{SnS} \mathrm{S}_{2}$, in comparison with a $30^{\circ}$ twisted $\mathrm{t}-\mathrm{SnS}_{2}$ bilayer.

and the incorporation of excess $\mathrm{S}$ into the growing $\mathrm{SnS}$ flakes are not activated, whereas $S$ rapidly desorbs at higher $T$, likely via the formation of weakly bound $\mathrm{S}_{\mathrm{x}}$ species (Supplementary Table 2). At intermediate $T$, here $280-320^{\circ} \mathrm{C}$, sulfur is available on the surface to spontaneously transform the growing $\mathrm{SnS}$ to twisted t-SnS ${ }_{2}$ (Supplementary Fig. 9). Note that the decomposition of the $\mathrm{SnS}_{2}$ surface layer via the thermally activated reaction $\mathrm{SnS}_{2} \rightarrow$ $\mathrm{SnS}+\mathrm{S}$ produces $\mathrm{SnS}$ and sulfur that are both mobile on the surface (Supplementary Fig. 3, Supplementary Fig. 7). Whereas the former adds to the deposited SnS, the adsorbed S contributes to the transformation of some of the SnS flakes into twisted $\mathrm{t}-\mathrm{SnS}_{2}$. The limited amount of sulfur released from the substrate implies that only a small fraction of the SnS flakes can be converted to $\mathrm{t}-\mathrm{SnS}_{2}$, as is indeed observed in Fig. 4. A higher yield of twisted $\mathrm{SnS}_{2}$ flakes may be obtained by supplying additional $\mathrm{S}$ from an external source. In this way, twisted dichalcogenide heterostructures can also realized on substrates that do not release substantial amounts of chalcogens (e.g., $\mathrm{MoS}_{2}, \mathrm{WS}_{2}$; see below).

AFM imaging confirms that the converted $\mathrm{t}-\mathrm{SnS}_{2}$ is indeed ultrathin. Figure $5 \mathrm{a}$ shows coexisting $\mathrm{SnS}$ and $\mathrm{t}-\mathrm{SnS}_{2}$ domains, where the latter are merely two atomic layers $\left(2 \mathrm{~L}^{\mathrm{SnS}_{2}}\right)$ thick (Fig. 5b). SnS and t-SnS $2 / \mathrm{SnS}_{2}$ are clearly distinguished via their surface potential, measured by KPFM (Fig. 5c, d). Generally, the potential $\phi^{\mathrm{SnS}}$ of SnS is higher than that of the surrounding $\mathrm{SnS}_{2}$ substrate, with $\Delta \phi=\phi^{\mathrm{SnS}}-\phi^{\mathrm{SnS} S_{2}} \cong+400 \mathrm{mV}$ measured by KPFM in air. Twisted $\mathrm{t}-\mathrm{SnS}_{2}$ domains show the same potential as the $\mathrm{SnS}_{2}$ substrate as expected due to their identical chemical nature and minimal potential shift due to twisted stacking. These findings are confirmed by local LEEM $I-V$ measurements of the surface potential in ultrahigh vacuum (UHV, see Supplementary Fig. 10 $)^{49}$. Pristine samples again show a large positive potential of SnS relative to the surrounding $\mathrm{SnS}_{2}$ but a negligible difference between $\mathrm{t}-\mathrm{SnS}_{2}$ and $\mathrm{SnS}_{2}$. For pristine $\mathrm{SnS}$ domains in UHV, $\Delta \phi>+1.0 \mathrm{~V}$ (Supplementary Fig. 10, Supplementary Fig. 11). Air exposure changes both $\phi^{\mathrm{SnS}}$ and $\phi^{\mathrm{SnS}}{ }_{2}$, reducing $\Delta \phi$ to $\sim 380 \mathrm{mV}$, consistent with the KPFM results. Annealing in UHV essentially recovers the pristine $\Delta \phi \sim+1.0 \mathrm{~V}$, consistent with adsorption of ambient species, strongly bound on $\mathrm{SnS}$ but weaker on $\mathrm{SnS}_{2}$ (as shown by $T$ for recovery of pristine potentials, $\mathrm{SnS}_{2}: \sim 200^{\circ} \mathrm{C}$; $\mathrm{SnS}: \sim 300^{\circ} \mathrm{C}$ ).

We find a strong preference for SnS nucleation on ultrathin $\mathrm{t}-\mathrm{SnS}_{2}$ domains during continued growth, which can give rise to a cyclic sequence of SnS growth and transformation to twisted $\mathrm{SnS}_{2}$. In situ microscopy illustrates this effect (Fig. 6). Starting with a $30^{\circ}$-rotated $\mathrm{t}-\mathrm{SnS}_{2} / \mathrm{SnS}_{2}$ twist heterostructure, further $\mathrm{SnS}$ deposition causes the t-SnS ${ }_{2}$ domain to expand laterally, implying a continued reaction of $\mathrm{SnS}$ to $\mathrm{SnS}_{2}$ (Fig. 6a, b). This suggests that the $\mathrm{t}-\mathrm{SnS}_{2}$ domains represent efficient sinks for adsorbed $\mathrm{SnS}$, which attaches to the edges, captures $\mathrm{S}$, and rapidly reacts to $\mathrm{SnS}_{2}$ at the microscopic level so that no $\mathrm{SnS}$ is detectable during realtime microscopy of the $\mathrm{t}-\mathrm{SnS}_{2}$ domain expansion. Ultimately, $\mathrm{SnS}$ nucleates either homogeneously or, as shown here, heterogeneously near the coalescence point of two $\mathrm{t}-\mathrm{SnS}_{2}$ domains (Fig. 6c; $450 \mathrm{~s}$ ). The subsequent evolution involves the spreading of $\mathrm{SnS}$ confined to $\mathrm{t}-\mathrm{SnS}_{2}$, followed by a renewed transformation to $\mathrm{SnS}_{2}$ (Fig. 6c; 490-530 s). Based on the characteristic contrast of $\mathrm{SnS}_{2}, \mathrm{SnS}$, and $\mathrm{t}-\mathrm{SnS}_{2}$, we find that $\mathrm{SnS}$ confined to t-SnS ${ }_{2}$ again undergoes a $30^{\circ}$ azimuthal rotation relative to the underlying lattice as it transforms to $\mathrm{SnS}_{2}$. As illustrated in Fig. 6d, this additional twist brings this new $\mathrm{SnS}_{2}$ layer back into azimuthal alignment with the substrate. In this way, alternating ultrathin $\mathrm{SnS}_{2}$ with $0^{\circ}$ and $30^{\circ}$ twist is formed, suggesting that complex heterostructures, e.g., periodic vertical vdW superlattices with multiple twisted interfaces, may be realized by direct growth

To demonstrate the generality of the concepts identified here, i.e., a strong tendency toward azimuthally aligned vdW epitaxy in nonisotypic $2 \mathrm{D}$ chalcogenide semiconductors and the ability of transforming an aligned intermediate to realize twisted vdW stacks-we performed additional growth experiments involving $\mathrm{SnS}$ vdW epitaxy on $\mathrm{MoS}_{2}$ (Fig. 7, Supplementary Fig. 12) and $\mathrm{WS}_{2}$ (Supplementary Fig. 13) substrates. On $\mathrm{MoS}_{2}$, SnS growth at $300^{\circ} \mathrm{C}$ produces ensembles of high-quality few-layer SnS flakes that expand to several $\mu \mathrm{m}$ lateral size (Supplementary Fig. 12) and exhibit a well-ordered layered morphology (Fig. 7a). Micro-LEED shows single-crystal diffraction patterns for both the $\mathrm{MoS}_{2}$ substrate (Fig. 7b) and the SnS flakes (Fig. 7c). Importantly, diffraction analysis shows the same azimuthal alignment for $\mathrm{SnS}$ on $\mathrm{MoS}_{2}$ as found for $\mathrm{SnS}$ on $\mathrm{SnS}_{2}$, namely the $(11)^{\mathrm{SnS}}$ reflection of $\mathrm{SnS}$ aligns with the $(10)^{\mathrm{MoS}_{2}}$ reflection of the $\mathrm{MoS}_{2}$ substrate. Likewise, growth on $\mathrm{WS}_{2}$ again locks the SnS into the same azimuthal registry with the substrate (Supplementary Fig. 13). While the in-plane orientation is fixed, the SnS flakes grow unstrained with their native inplane lattice parameters on the different substrates.

In contrast to growth on $\mathrm{SnS}_{2}$, where some of the SnS flakes transform to $\mathrm{t}-\mathrm{SnS}_{2}$, such a spontaneous transformation is not observed during growth on $\mathrm{MoS}_{2}$ or $\mathrm{WS}_{2}$, consistent with the absence of their thermal decomposition into stable Mo or $\mathrm{W}$ 
a

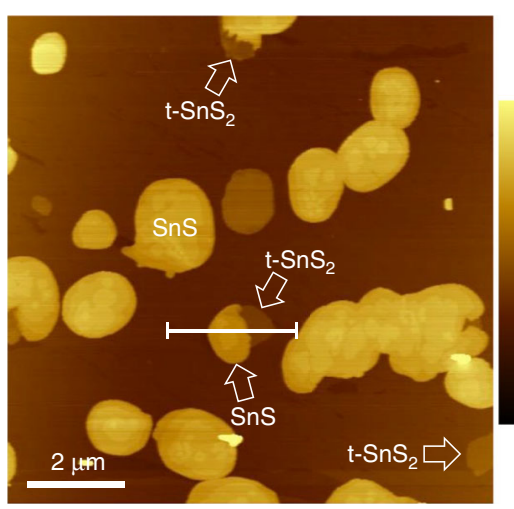

C

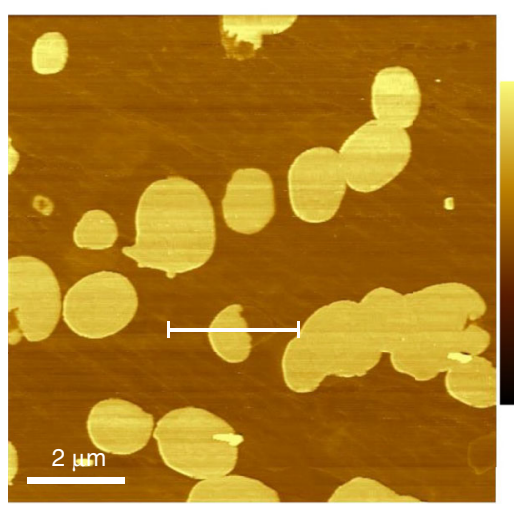

b

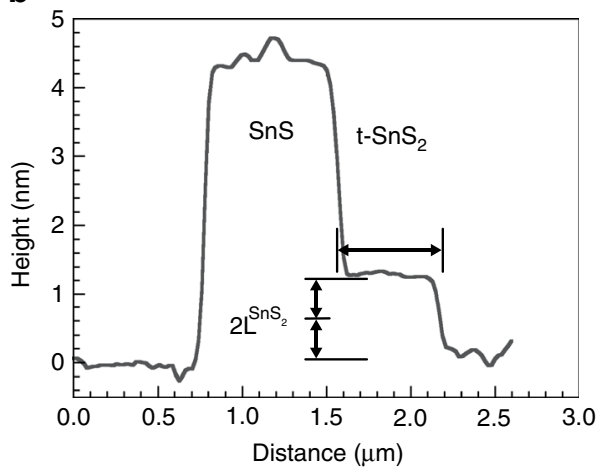

d

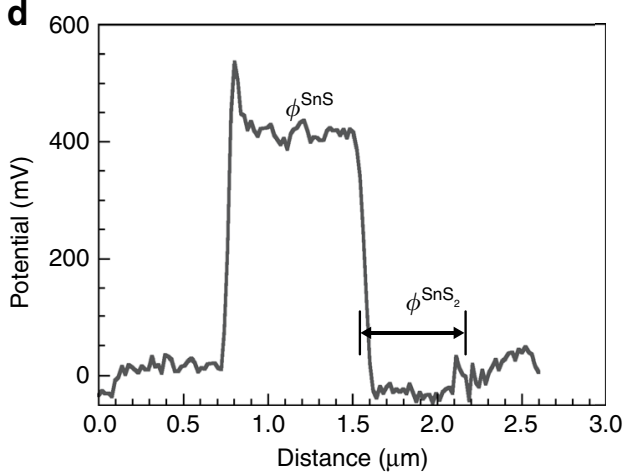

Fig. 5 Ultrathin self-organized t-SnS/ $\mathrm{SnS}_{2}$ twist heterostructures. a AFM image showing coexisting $\mathrm{SnS}_{\mathrm{SnS}}$ and twisted t-SnS $2 / \mathrm{SnS}_{2}$. Color scale: $15 \mathrm{~nm}$. b Height profile along the line marked in $\mathbf{a}$, identifying the $\mathrm{t}-\mathrm{SnS}_{2}$ as a bilayer. c KPFM surface potential map of the area shown in a. Color scale: $1.0 \mathrm{~V}$. d Potential profile along the same line trace as $\mathbf{b}$, as marked in the maps in panels $\mathbf{a}$. and $\mathbf{c}$. In contrast to SnS, which shows a large potential difference, $\phi^{\mathrm{SnS}}-\phi^{\mathrm{SnS}} \cong+400 \mathrm{mV}$, relative to the surrounding $\mathrm{SnS}_{2}$, the surface potential of the $30^{\circ}$ twisted t-SnS 2 is indistinguishable from that of the $\mathrm{SnS}_{2}$ substrate. Surface potential measurements can thus unambiguously distinguish $\mathrm{t}-\mathrm{SnS}_{2}$ from $\mathrm{SnS}$.
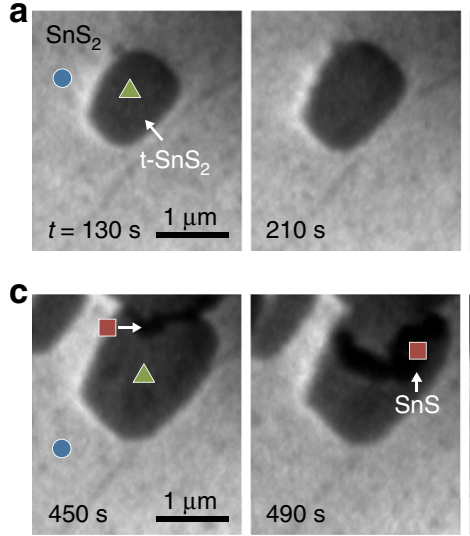
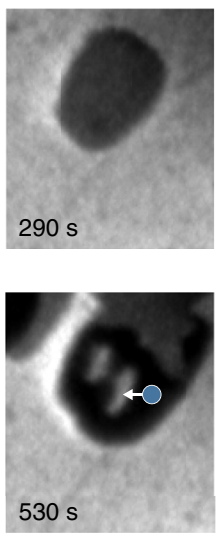
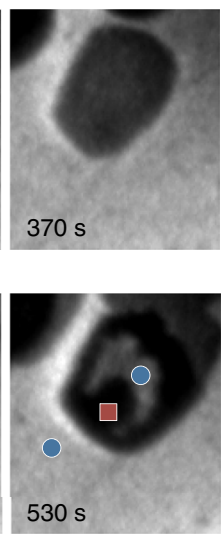

b

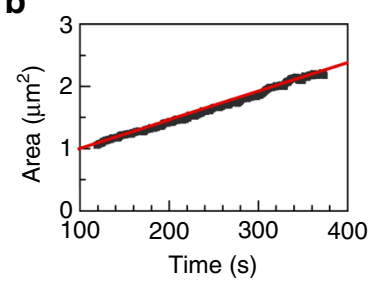

d

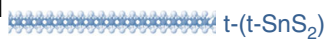

$40^{\circ}$

Y $30^{\circ}$

$\Psi 30^{\circ}{ }^{t-S n S_{2}}$

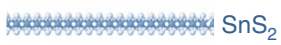

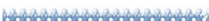

Fig. 6 Cyclic twisted $\mathrm{SnS}_{2}$ growth and incipient SnS-mediated vertical twist-superlattices. a Real-time image sequence during continued deposition of SnS (following Fig. 3), showing the lateral expansion of the $\mathrm{t}-\mathrm{SnS}_{2}$ domain. Imaging electron energy: $E=5.0 \mathrm{eV}$. b Analysis of the growth in projected area of the $\mathrm{t}-\mathrm{SnS}_{2}$ domain. $\mathbf{c} \mathrm{SnS}$ nucleation and spreading across the $\mathrm{t}-\mathrm{SnS}_{2}$ footprint, followed by transformation to $\mathrm{SnS}_{2}$. d Schematic showing the twisted van der Waals stack resulting from cyclic $\mathrm{SnS}$ growth and transformation to $\mathrm{SnS}_{2}$.

monochalcogenides. However, conversion of the deposited SnS to $\mathrm{SnS}_{2}$ can be realized by post-growth reaction with sulfur (see Methods), as shown by Raman spectroscopy analysis in Fig. $7 \mathrm{~d}-\mathrm{i}$. Figure 7d, e shows Raman linescans of as-grown few-layer and ultrathin SnS flakes on $\mathrm{MoS}_{2}$. Uniform modes across the SnS flakes confirm their single-crystallinity ${ }^{48}$. Representative spectra show the characteristic $B_{3 \mathrm{~g}}$ and $A_{\mathrm{g}}$ modes of $\mathrm{SnS}$ along with intense $E_{2 \mathrm{~g}}^{l}$ and $A_{1 \mathrm{~g}}$ peaks of the $\mathrm{MoS}_{2}$ substrate (Fig. $\left.7 \mathrm{f}, \mathrm{g}\right)^{50}$. In the as-grown flakes, the most intense vibrational mode of $\mathrm{SnS}_{2}$ $\left(A_{1 g}, \sim 311 \mathrm{~cm}^{-1}\right)^{36}$ is not detectable. Figure $7 \mathrm{~h}$, i shows Raman spectra from flakes on the same sample following a 2-hour exposure to sulfur vapor at $370^{\circ} \mathrm{C}$. After sulfurization, few-layer flakes show the appearance of the $\mathrm{SnS}_{2} A_{1 \mathrm{~g}}$ peak with intensity similar to that of the SnS modes (Fig. 7h), indicating a partial 
a

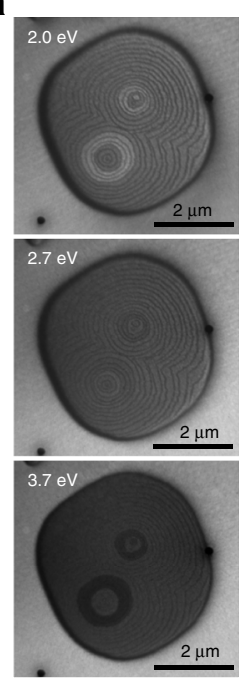

b

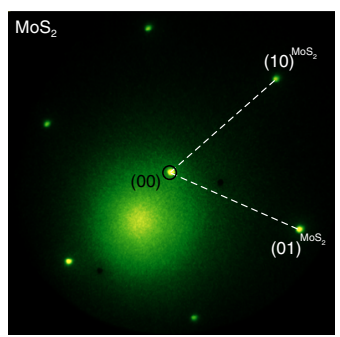

c

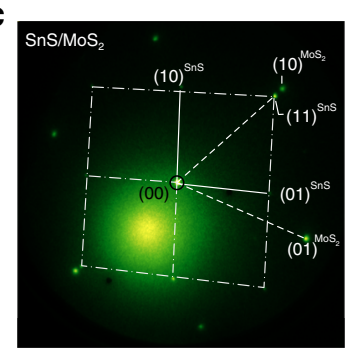

d
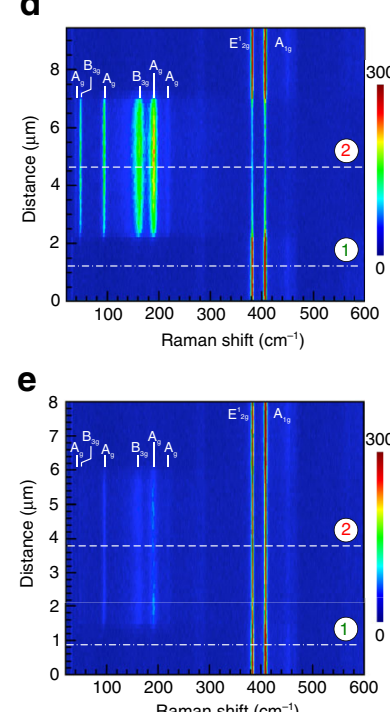

f

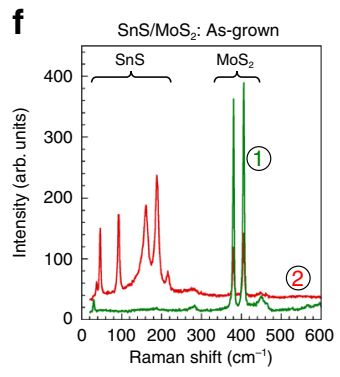

g

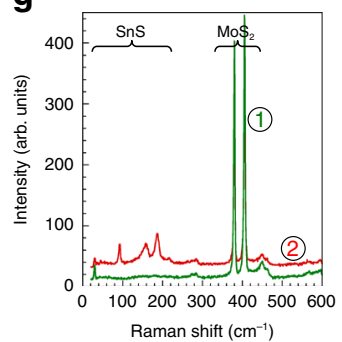

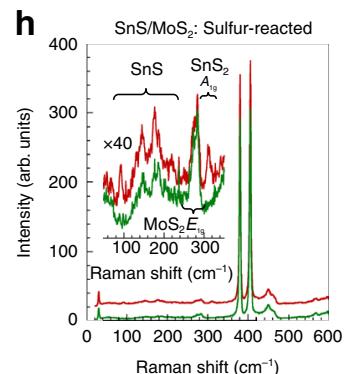

i

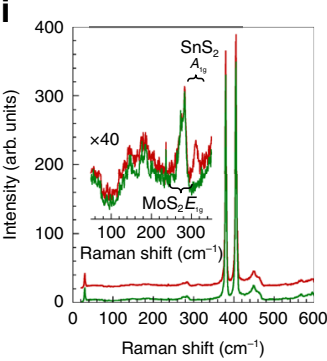

Fig. 7 Extension to other materials systems $-\mathrm{MoS}_{2}$. a LEEM images of a few-layer SnS domain grown at $T=300^{\circ} \mathrm{C}$ on a $\mathrm{MoS}_{2}$ substrate, imaged at different electron energies, E. b Micro-LEED pattern of the trigonal $\mathrm{MoS}_{2}$ substrate. c Micro-LEED pattern of SnS grown on MoS 2 . Note the two sets of diffraction spots, originating from SnS and $\mathrm{MoS}_{2}$, respectively, and the azimuthal alignment of the $(11)^{\mathrm{SnS}}$ reflection of SnS and the $(10)^{\mathrm{MoS}_{2}}$ reflection of $\mathrm{MoS}_{2}$. Both lattices adopt their bulk lattice constants during the vdW epitaxy. d Raman linescan of a thicker few-layer SnS flake with $\sim 5 \mu \mathrm{m}$ lateral size on $\mathrm{MoS}_{2}$, with the major Raman-active modes of both materials identified. e Raman linescan of an ultrathin SnS flake with $\sim 5 \mu \mathrm{m}$ lateral size on MoS 2 . f Raman spectra of the few-layer SnS flake (red) and of the nearby $\mathrm{MoS}_{2}$ substrate (green), extracted at locations shown in $\mathbf{d}$. $\mathbf{g}$ Raman spectra of the ultrathin SnS flake (red) and of the nearby $\mathrm{MoS}_{2}$ substrate (green), extracted at locations shown in e. h Raman spectra of a few-layer SnS flake (red) and of the MoS 2 substrate (green), following reaction with sulfur at $370{ }^{\circ} \mathrm{C}$ for $2 \mathrm{~h}$. The inset shows the intensity of the main SnS Raman modes, along with an additional peak at $311 \mathrm{~cm}^{-1}$ due to the $A_{1 \mathrm{~g}}$ mode of $\mathrm{SnS}_{2}$. i Raman spectra of an ultrathin $\mathrm{SnS}$ flake (red) and of the $\mathrm{MoS}_{2}$ substrate (green), following reaction with sulfur at $370^{\circ} \mathrm{C}$ for $2 \mathrm{~h}$. The inset shows the absence of $\mathrm{SnS}$ Raman modes and a peak at $311 \mathrm{~cm}^{-1}$ due to the $A_{1 \mathrm{~g}}$ mode of $\mathrm{SnS}_{2}$, indicating complete conversion of the ultrathin $\mathrm{SnS}$ to $\mathrm{SnS}_{2}$.

transformation to $\mathrm{SnS}_{2}$ during annealing in S-vapor. For ultrathin sulfurized flakes, the SnS vibrational modes are no longer detectable but are replaced by the $\mathrm{SnS}_{2} A_{1 \mathrm{~g}}$ mode, indicating a complete transformation of these $\mathrm{SnS}$ flakes to $\mathrm{SnS}_{2}$.

\section{Conclusions}

From our combined findings, we can draw several conclusions. Firstly, azimuthally aligned vdW growth appears to be widespread, even for non-isotypic crystals such as orthorhombic SnS on trigonal $\mathrm{SnS}_{2}, \mathrm{MoS}_{2}$, and $\mathrm{WS}_{2}$ substrates. In cases where the support can release significant amounts of sulfur at the growth temperature, as is the case for $\mathrm{SnS}_{2}$ substrates, ultrathin aligned intermediate crystals can spontaneously transform to form twisted heterostructures. On substrates with limited sulfur release, a two-step process with post-growth sulfurization can achieve the same result. Our observations also suggest that exposure to additional sulfur may be used to increase the fraction of SnS flakes that transform to $\mathrm{t}-\mathrm{SnS}_{2}$ during growth on $\mathrm{SnS}_{2}$ substrates. Finally, we found indications that the nucleation and transformation of an azimuthally aligned intermediate crystal phase may also govern the growth on non-chalcogenide substrates, if sulfur is provided in the vapor phase. As shown in Supplementary Figure 14, vapor transport growth of $\mathrm{SnS}$ on mica substrates with exposure to sulfur gives rise to two populations of oriented $\mathrm{SnS}_{2}$ flakes: A majority phase ( $\sim 2 \%$ of all flakes) and a minority phase $(\sim 18 \%)$ rotated by $30^{\circ}$. This again supports a growth mechanism in which aligned $\mathrm{SnS}$ crystals ultimately transform into $30^{\circ}$ rotated $\mathrm{t}-\mathrm{SnS}_{2}$. While the work reported here focused on transformations between $2 \mathrm{D} \mathrm{SnS}$ and $\mathrm{t}-\mathrm{SnS}_{2}$ crystals to realize the bottom-up synthesis of twisted vdW heterostructures, our results raise the possibility of using the sulfurization of $3 \mathrm{D}$ crystals, e.g., thin metal or metal oxide templates for forming twisted chalcogenide heterostructures. Future work needs to show if such 3D intermediates can be grown in a fixed azimuthal orientation with a vdW substrate, and if the azimuthal orientation of the final chalcogenide phase after transformation with sulfur will again be defined by the intermediate rather than the substrate.

\section{Methods}

Low-energy electron microscopy and micro-LEED of SnS growth on vdW substrates. High-quality $\mathrm{SnS}_{2}$ single crystals synthesized by the vertical Bridgman method were used as substrates. Additional layered supports included bulk $\mathrm{MoS}_{2}$ (extracted from natural minerals) and $\mathrm{WS}_{2}$ (synthetic, 2D Semiconductors). Prior to growth the layered substrates were mechanically cleaved and degassed at $\sim 300^{\circ} \mathrm{C}$ in ultrahigh vacuum (UHV). SnS was congruently evaporated from SnS powder (99.99\%, Sigma-Aldrich) using a custom-built miniature Knudsen cell heated to $400-450{ }^{\circ} \mathrm{C}$ while observing the resulting surface processes in real time by bright-field LEEM. In situ LEEM, Micro-LEED, and other complementary measurements were performed in a modified Elmitec LEEM III microscope that allows observations at variable temperature in UHV (base pressure $2 \times 10^{-10}$ Torr) and during sample exposure to gases or vapors (notably chalcogens or chalcogenides) with $\sim 6 \mathrm{~nm}$ lateral and monolayer height resolution. Sample temperatures in

LEEM were measured using a W-Re thermocouple spot-welded onto the sample support. Real-time image sequences were acquired at a rate of 1 frame per second and recorded at $1024 \times 1024$ pixels. Micro-LEED was performed in selected areas with $\sim 1 \mu \mathrm{m}$ lateral size. LEEM I-V data were acquired in real space with the full spatial resolution of the microscope; reported curves represent averages over areas with $200-400 \mathrm{~nm}$ lateral size.

Post-growth sulfurization. SnS samples grown on $\mathrm{MoS}_{2}$ substrates were exposed to sulfur vapor in a separate quartz reactor implemented in a single-zone tube furnace with an additional external heating zone for sulfur. Sulfur powder (99.9995\%, Alfa Aesar) was loaded into a quartz boat and as-grown SnS flakes on $\mathrm{MoS}_{2}$ were positioned in the center of the heating zone of the furnace. Following evacuation of the reactor a carrier gas mixture of $\mathrm{Ar}$ and $\mathrm{H}_{2}$ (ratio 98:2) was introduced at a flow rate of $50 \mathrm{sccm}$ and a pressure of 76 Torr. The sulfur reservoir was heated to $80^{\circ} \mathrm{C}$ (vapor pressure $\sim 4 \times 10^{-4}$ Torr) and sulfurization was carried out at a sample temperature of $370^{\circ} \mathrm{C}$ for $2 \mathrm{~h}$, followed by natural cooling to room temperature. 
Ex-situ measurements. AFM, phase mapping, and KPFM were carried out in tapping mode in air using a Veeco Multimode microscope with commercial SiN cantilevers or probes coated with thin metallic $(\mathrm{Ru}, \sim 10 \mathrm{~nm})$ films. Raman spectroscopy was performed at room temperature in air in a Horiba Xplora Plus Raman microscope using a 100x objective at excitation wavelength of $532 \mathrm{~nm}$ and laser power of $0.168 \mu \mathrm{W}$. Computational methods are reported in the Supplementary Methods.

\section{Data availability}

All relevant data are available upon reasonable request from the corresponding author. The source data underlying Figs. $2 \mathrm{c}$ and $2 \mathrm{e}$ are provided as a Source Data file.

Received: 31 May 2019; Accepted: 7 November 2019;

Published online: 04 December 2019

\section{References}

1. Geim, A. K. \& Grigorieva, I. V. Van der Waals heterostructures. Nature 499, 419 (2013).

2. Koma, A., Sunouchi, K. \& Miyajima, T. Fabrication and characterization of heterostructures with subnanometer thickness. Microelectron. Eng. 2, 129-136 (1984).

3. Ohuchi, F. S., Parkinson, B. A., Ueno, K. \& Koma, A. van der Waals epitaxial growth and characterization of $\mathrm{MoSe}_{2}$ thin films on $\mathrm{SnS}_{2}$. J. Appl. Phys. 68, 2168-2175 (1990).

4. Cao, Y. et al. Correlated insulator behaviour at half-filling in magic-angle graphene superlattices. Nature 556, 80 (2018).

5. Cao, Y. et al. Unconventional superconductivity in magic-angle graphene superlattices. Nature 556, 43 (2018).

6. Liu, K. et al. Evolution of interlayer coupling in twisted molybdenum disulfide bilayers. Nat. Commun. 5, 4966 (2014).

7. Yeh, P.-C. et al. Direct measurement of the tunable electronic structure of bilayer $\mathrm{MoS}_{2}$ by interlayer twist. Nano Lett. 16, 953-959 (2016).

8. Carr, S. et al. Twistronics: manipulating the electronic properties of twodimensional layered structures through their twist angle. Phys. Rev. B 95 , 075420 (2017).

9. Yu, H., Wang, Y., Tong, Q., Xu, X. \& Yao, W. Anomalous light cones and valley optical selection rules of interlayer excitons in twisted heterobilayers. Phys. Rev. Lett. 115, 187002 (2015).

10. Jin, C. et al. Observation of moiré excitons in $\mathrm{WSe}_{2} / \mathrm{WS}_{2}$ heterostructure superlattices. Nature 567, 76-80 (2019).

11. Seyler, K. L. et al. Signatures of moiré-trapped valley excitons in $\mathrm{MoSe}_{2} / \mathrm{WSe}_{2}$ heterobilayers. Nature 567, 66-70 (2019).

12. Tran, K. et al. Evidence for moiré excitons in van der Waals heterostructures. Nature 567, 71-75 (2019).

13. Dean, C. et al. Graphene based heterostructures. Solid State Commun. 152, 1275-1282 (2012).

14. Kim, K. et al. van der Waals heterostructures with high accuracy rotational alignment. Nano Lett. 16, 1989-1995 (2016).

15. Kang, K. et al. Layer-by-layer assembly of two-dimensional materials into wafer-scale heterostructures. Nature 550, 229 (2017).

16. Mishchenko, A. et al. Twist-controlled resonant tunnelling in graphene/boron nitride/graphene heterostructures. Nat. Nanotechnol. 9, 808-813 (2014).

17. Greenaway, M. T. et al. Resonant tunnelling between the chiral Landau states of twisted graphene lattices. Nat. Phys. 11, 1057 (2015).

18. Cao, Y. et al. Superlattice-induced insulating states and valley-protected orbits in twisted bilayer graphene. Phys. Rev. Lett. 117, 116804 (2016).

19. Ribeiro-Palau, R. et al. Twistable electronics with dynamically rotatable heterostructures. Science 361, 690-693 (2018).

20. Fang, H. et al. Strong interlayer coupling in van der Waals heterostructures built from single-layer chalcogenides. Proc. Natl Acad. Sci. USA 111, 6198-6202 (2014)

21. Gong, Y. et al. Two-step growth of two-dimensional WSe $2 / \mathrm{MoSe}_{2}$ heterostructures. Nano Lett. 15, 6135-6141 (2015).

22. Gong, Y. et al. Vertical and in-plane heterostructures from $\mathrm{WS}_{2} / \mathrm{MoS}_{2}$ monolayers. Nat. Mater. 13, 1135 (2014).

23. Sutter, P. \& Sutter, E. Growth mechanisms of anisotropic layered group IV chalcogenides on van der Waals substrates for energy conversion applications. ACS Appl. Nano Mater. 1, 3026-3034 (2018).

24. Woods, J. M. et al. One-step synthesis of $\mathrm{MoS}_{2} / \mathrm{WS}_{2}$ layered heterostructures and catalytic activity of defective transition metal dichalcogenide films. ACS Nano 10, 2004-2009 (2016).

25. Xue, Y. et al. Scalable production of a few-layer $\mathrm{MoS}_{2} / \mathrm{WS}_{2}$ vertical heterojunction array and its application for photodetectors. ACS Nano 10, 573-580 (2016).
26. Zhang, C. et al. Interlayer couplings, Moiré patterns, and $2 \mathrm{D}$ electronic superlattices in $\mathrm{MoS}_{2} / \mathrm{WSe}_{2}$ hetero-bilayers. Sci. Adv. 3, el601459 (2017).

27. Liu, X. et al. Rotationally commensurate growth of $\mathrm{MoS}_{2}$ on epitaxial graphene. ACS Nano 10, 1067-1075 (2016)

28. Tsoutsou, D. et al. Epitaxial $2 \mathrm{D} \mathrm{MoSe} 2\left(\mathrm{HfSe}_{2}\right)$ semiconductor/2D TaSe $\mathrm{Metal}_{2}$ van der waals heterostructures. ACS Appl. Mater. Interfaces 8, 1836-1841 (2016).

29. Zhang, X. et al. Transition metal dichalcogenides bilayer single crystals by reverse-flow chemical vapor epitaxy. Nat. Commun. 10, 598 (2019).

30. Sutter, P., Wimer, S. \& Sutter, E. Chiral twisted van der Waals nanowires. Nature 570, 354-357 (2019).

31. Ahn, S. J. et al. Dirac electrons in a dodecagonal graphene quasicrystal. Science 361, 782-786 (2018).

32. Sousa, M. G., da Cunha, A. F. \& Fernandes, P. A. Annealing of RF-magnetron sputtered $\mathrm{SnS}_{2}$ precursors as a new route for single phase $\mathrm{SnS}$ thin films. J. Alloy. Compd. 592, 80-85 (2014).

33. Sutter, E. et al. Electron-beam induced transformations of layered tin dichalcogenides. Nano Lett. 16, 4410-4416 (2016)

34. Kim, J. H. et al. Plasma-induced phase transformation of $\mathrm{SnS}_{2}$ to SnS. Sci. Rep. 8, 10284 (2018)

35. $\mathrm{Li}, \mathrm{M}$. et al. One-step CVD fabrication and optoelectronic properties of $\mathrm{SnS}_{2}$ SnS vertical heterostructures. Inorg. Chem. Front. 5, 1828-1835 (2018).

36. Huang, Y. et al. Tin disulfide-an emerging layered metal dichalcogenide semiconductor: materials properties and device characteristics. ACS Nano 8 , 10743-10755 (2014)

37. Hazen, R. M. \& Finger, L. W. The crystal structures and compressibilities of layer minerals at high pressure. I. $\mathrm{SnS}_{2}$, berndtite. Am. Mineralogist 63, 289-292 (1978).

38. Wiedemeier, H., Georg, H. \& von Schnering, G. Refinement of the structures of GeS, GeSe, SnS and SnSe. Z. f.ür. Kristallographie-Crystalline Mater. 148, 295-304. (1978)

39. Fei, R., Kang, W. \& Yang, L. Ferroelectricity and phase transitions in monolayer group-IV monochalcogenides. Phys. Rev. Lett. 117, 097601 (2016)

40. Fei, R., Li, W., Li, J. \& Yang, L. Giant piezoelectricity of monolayer group IV monochalcogenides: SnSe, SnS, GeSe, and GeS. Appl. Phys. Lett. 107, 173104 (2015).

41. Wu, M. \& Zeng, X. C. Intrinsic ferroelasticity and/or multiferroicity in twodimensional phosphorene and phosphorene analogues. Nano Lett. 16, 3236-3241 (2016)

42. Haleoot, R. et al. Photostrictive two-dimensional materials in the monochalcogenide family. Phys. Rev. Lett. 118, 227401 (2017)

43. Tian, Z., Guo, C., Zhao, M., Li, R. \& Xue, J. Two-dimensional SnS: a phosphorene analogue with strong in-plane electronic anisotropy. ACS Nano 11, 2219-2226 (2017)

44. Sutter, E., Wang, J. \& Sutter, P. Nanoparticle-templated thickness controlled growth, thermal stability, and decomposition of ultrathin tin sulfide plates. Chem. Mater. 31, 2563-2570 (2019).

45. Stampfli, P. A dodecagonal quasi-periodic lattice in 2 dimensions. Helvetica Phys. Acta 59, 1260-1263 (1986).

46. Yang, Y. B. et al. Large single crystal SnS2 flakes synthesized from coevaporation of Sn and S. Cryst. Growth Des. 16, 961-973 (2016)

47. Kerr, J. A. CRC Handbook of Chemistry and Physics, "Bond Dissociation Energies". (CRC Press, Boca Raton, FL, 2005).

48. Sutter, P., Wang, J. \& Sutter, E. Wrap-around core-shell heterostructures of layered crystals. Adv. Mater. 31, 1902166 (2019)

49. Sutter, P. \& Sutter, E. Microscopy of graphene growth, processing, and properties. Adv. Funct. Mater. 23, 2617-2634 (2013).

50. Li, H. et al. From bulk to monolayer $\mathrm{MoS}_{2}$ : evolution of raman scattering. $A d v$. Funct. Mater. 22, 1385-1390 (2012).

\section{Acknowledgements}

This work was supported by the U.S. Department of Energy, Office of Science, Basic Energy Sciences, under Award No. DE-SC0016343. The authors acknowledge J. Wang for technical support. R.I. and H.-P.K. acknowledge Academy of Finland for support under Academy research fellow grant No. 311058 and CSC-IT Center for Science Ltd. for the computing resources.

\section{Author contributions}

P.S. and E.S. designed the study, carried out the experiments, and analysed the data. R.I and H.-P.K. performed the theoretical calculations. B.A.P. contributed high-quality $\mathrm{SnS}_{2}$ single crystals. P.S. and E.S. wrote the paper, and all authors commented on the manuscript.

\section{Competing interests}

The authors declare no competing interests. 


\section{Additional information}

Supplementary information is available for this paper at https://doi.org/10.1038/s41467019-13488-5.

Correspondence and requests for materials should be addressed to P.S.

Peer review information Nature Communications thanks Joung Real Ahn, Shaoqing Xiao and the other anonymous reviewer(s) for their contribution to the peer review of this work

Reprints and permission information is available at http://www.nature.com/reprints

Publisher's note Springer Nature remains neutral with regard to jurisdictional claims in published maps and institutional affiliations. (c) (i) Open Access This article is licensed under a Creative Commons Attribution 4.0 International License, which permits use, sharing, adaptation, distribution and reproduction in any medium or format, as long as you give appropriate credit to the original author(s) and the source, provide a link to the Creative Commons license, and indicate if changes were made. The images or other third party material in this article are included in the article's Creative Commons license, unless indicated otherwise in a credit line to the material. If material is not included in the article's Creative Commons license and your intended use is not permitted by statutory regulation or exceeds the permitted use, you will need to obtain permission directly from the copyright holder. To view a copy of this license, visit http://creativecommons.org/ licenses/by/4.0/.

(C) The Author(s) 2019 